\title{
Experimental sensitivity analysis and control of thermoacoustic systems
}

\author{
Georgios $\operatorname{Rigas}^{1} \dagger$, Nicholas P. Jamieson ${ }^{1}$, Larry K. B. Li ${ }^{2}$ and \\ Matthew P. Juniper ${ }^{1}$ \\ ${ }^{1}$ Department of Engineering, University of Cambridge, Trumpington Street, Cambridge, CB2 \\ $1 \mathrm{PZ}, \mathrm{UK}$ \\ ${ }^{2}$ Department of Mechanical and Aerospace Engineering, The Hong Kong University of Science \\ and Technology, Clear Water Bay, Kowloon, Hong Kong
}

(Received $\mathrm{xx}$; revised $\mathrm{xx}$; accepted $\mathrm{xx}$ )

In this paper, we report the results of an experimental sensitivity analysis on a thermoacoustic system, an electrically-heated Rijke tube. We measure the change of the linear stability characteristics of the system, quantified as shifts in the growth rate and oscillation frequency, that is caused by the introduction of a passive control device. The control device is a mesh, which causes drag in the system. The rate of growth is slow, so the growth rate and frequency can be measured very accurately over many hundred cycles in the linear regime with and without control. These measurements agree qualitatively well with the theoretical predictions from adjoint-based methods of Magri \& Juniper (JFM 2013, 719, 183-202). This agreement supports the use of adjoint methods for the development and implementation of control strategies for more complex thermoacoustic systems.

Key words:

\section{Introduction}

In many combustion systems, there exist high-amplitude pressure oscillations whose frequency ranges are close to those of the natural acoustic modes of the system. These are known as thermoacoustic oscillations and arise from the feedback loop between acoustic waves and unsteady heat release if the latter occurs sufficiently in phase with unsteady pressure. The oscillations can threaten the operability and reliability of combustion systems by increasing the risks of thrust oscillations, mechanical vibration, excessive thermal and mechanical loading, resulting in decreased efficiency and ultimately system failure (Lieuwen \& Yang 2005).

In this paper, we investigate the passive control of a simple thermoacoustic system, an electrically-heated Rijke tube, via a drag device placed downstream of the heater. The main novelty is that we carefully measure and compare experimental results with theoretical predictions from adjoint sensitivity analysis. The findings suggest that adjointbased methods can provide industry with a valuable tool for developing optimal control strategies for more complex thermoacoustic systems. 


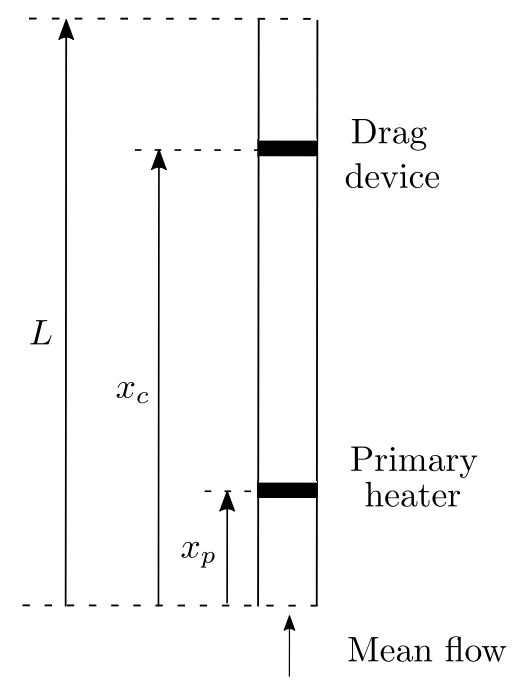

FiguRE 1. Rijke tube layout and notation. Thermoacoustic oscillations can be excited because acoustic velocity fluctuations cause heat release fluctuations at the primary electrical heater. If the primary heater is in the upstream half of the tube then higher heat release is sufficiently in phase with higher pressure that thermal energy is converted to acoustic energy over a cycle, making the system unstable. A mesh is used as a passive control device here, which causes drag in the system.

\subsection{Rijke tube and control}

The Rijke tube, first proposed by Rijke (1859), is a simple experiment through which thermoacoustic phenomena can be studied (Raun et al. 1993). The notation we use throughout this paper is given in figure 1 where $L$ is the length of the tube, $x_{p}$ is the distance of the electrical heater from the inlet, and $x_{c}$ is the distance of the drag device from the inlet.

To a reasonable approximation, the pressure fluctuation at the open ends of the Rijke tube is zero. Therefore the fundamental mode has a node at both ends and an anti-node in the middle. The acoustic velocity has a node at the center and two anti-nodes at either end. During the compression phase, the acoustic velocity is directed towards the centre of the tube. When the heater is placed at $x_{p} / L=0.25$, the heater experiences a higher acoustic velocity and therefore a higher heat transfer. There is a small time delay between the change in velocity and the consequent change in heat transfer, which causes the unsteady heat release to be slightly in phase with the acoustic pressure, resulting in the excitation of thermoacoustic oscillations (Rayleigh 1878).

The control methods used to regulate thermoacoustic oscillations can be divided into two categories: passive and active (McManus et al. 1993; Candel 2002; Dowling \& Morgans 2005). Passive control can be achieved by modifying the design or additive devices, such as Helmholtz resonators. A famous example of this was demonstrated in the development for the F1 engine of the Saturn V rocket used in the Apollo space missions (Culick 1988). Some of the earliest papers to report the passive control of thermoacoustic oscillations in a Rijke tube are by Katto \& Sajiki (1977) and Sreenivasan et al. (1985). These studies showed that oscillations present in a flame-driven Rijke tube

$\dagger$ Email address for correspondence: gr379@.cam.ac.uk 
can be suppressed by introducing a control heater downstream of the primary heater at certain positions.

\subsection{Sensitivity analysis and adjoint methods in thermoacoustics}

Sensitivity analysis typically quantifies (i) the sensitivity of each mode to internal feedback, known here as the structural sensitivity, achieved by introducing a passive device or a steady fixed forced signal, and, (ii) the sensitivity of each mode to changes in the base state, known here as the base-state sensitivity (Magri \& Juniper 2013), achieved by design modifications to the system. Further information can be found in the recent reviews of Sipp et al. (2010), Luchini \& Bottaro (2014) and Schmid \& Brandt (2014). Sensitivity analysis is usually performed via the finite difference approach. However, this method is computationally expensive and prone to numerical error. Given these limitations, adjoint methods have been found to offer a more efficient and accurate means of conducting sensitivity and receptivity analyses. The application of adjoint methods to sensitivity analysis in thermoacoustics has been developed over the past few years (Magri \& Juniper 2013, 2014a,b).

The study by Magri \& Juniper (2013) is the most relevant as the experimental set-up utilised in this paper consists of a Rijke tube with a gauze heater acting as the acoustically compact heat source. Magri \& Juniper (2013) examined the stability of the Rijke tube around a base flow in the limit of low Mach number, Ma $\ll 1$. This condition was met in our experiments as the measured bulk mean flow was less than $1 \mathrm{~m} / \mathrm{s}$, corresponding to a Mach number of less than 0.003 . The unsteady heat release from the hot wire was modelled with a generalised version of King's Law, and uniform temperature was assumed throughout the tube. Using adjoint methods, they calculated how the linear growth rate and frequency of thermoacoustic oscillations change when a passive control device was introduced to the system. They found that the growth rate of oscillation is most sensitive to a feedback mechanism that is proportional to the velocity and that forces the momentum equation. This type of feedback could be physically implemented in the form of a mesh, which forces the momentum equation in the opposite direction to the velocity by inducing a drag force. The authors also found that the passive device has the largest effect when placed at the ends of the Rijke tube. In this paper we experimentally investigate their findings.

\section{Experimental set-up}

Experiments were conducted on a $1 \mathrm{~m}$ long stainless steel vertical Rijke tube with an internal diameter of $47.4 \mathrm{~mm}$ and a wall thickness of $1.7 \mathrm{~mm}$. Two identical heaters were used. The primary heater was attached to two rods and held in place at $x_{p} / L=0.25$, the optimal position for exciting thermoacoustic oscillations (Saito 1965). It was powered by a 640 Watt EA Elektro-Automatik EA-PSI 5080-20 A DC programmable power supply. The secondary heater was used as a passive drag device with no power input. The passive drag device was attached to an automated digital height gauge at the top of the Rijke tube, enabling it to be traversed with an accuracy of $\pm 0.01 \mathrm{~mm}$.

A Brüel Kjaer condenser type 2619 microphone with a sensitivity of $11.4 \mathrm{mV} \mathrm{Pa}{ }^{-1}$ was used to measure pressure fluctuations. The microphone was angled at $45^{\circ}$ and placed $55 \mathrm{~mm}$ from the bottom end of the tube. The raw pressure signal was sampled at 10 $\mathrm{kHz}$, much higher than the anticipated frequencies of the thermoacoustic oscillations, approximately $190 \mathrm{~Hz}$. Data was acquired via a National Instruments BNC-2110 DAQ device using LabVIEW. Two type-K thermocouples, with an accuracy of $\pm 1 \mathrm{~K}$, were used to measure the air flow temperature at the inlet and outlet of the Rijke tube. Five type-K 


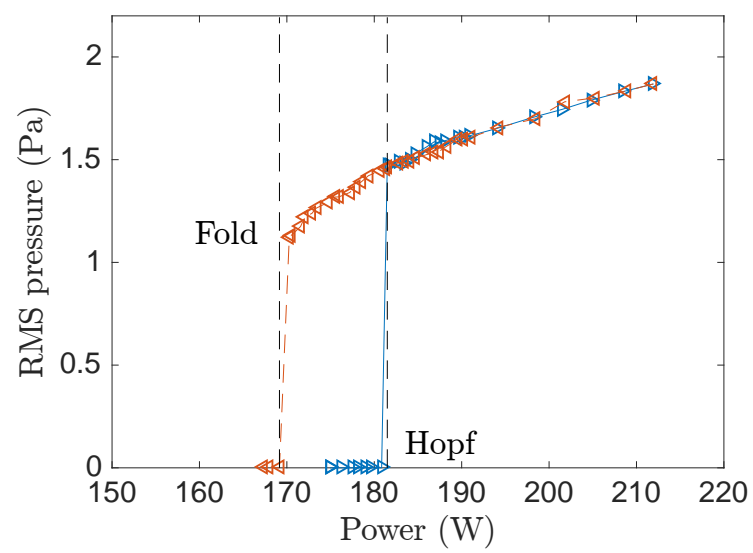

FiguRE 2. Bifurcation diagram obtained experimentally in the Rijke tube. The system becomes unstable through a subcritical Hopf bifurcation. The forward path (right-pointing triangles) shows the position of the Hopf point, and the backward path (left-pointing triangles) shows the position of the fold point.

thermocouples were attached to the outside of the Rijke tube, at $x / L=0.05,0.25,0.55$, 0.75 and 0.95 , to monitor the surface temperature of the tube. The temperature data was sampled at a frequency of $1 \mathrm{~Hz}$ and logged with an Omega TC-08 DAQ.

\section{Baseline}

The baseline experiments were performed without the passive drag device. The electric heater was fixed at $x_{p} / L=0.25$ and the power supplied to it was used as the control parameter.

Figure 2 shows the steady-state root-mean-square amplitude of the pressure signal as a function of the heater power. The heater power was increased in $0.8 \mathrm{~W}$ increments until the Hopf point was found (forward path), and then decreased in $0.8 \mathrm{~W}$ increments until the fold point was found (backward path). When the Hopf point was found, the pressure signal grew in amplitude leading to a stable limit-cycle. When the fold point was found, the system returned to a stable fixed point. A hysteresis region of approximately $25 \mathrm{~W}$ wide exists between the Hopf and fold points, characteristic of the subcritical bifurcations observed in similar thermoacoustic systems (Crocco et al. 1969; Matveev 2003; Mariappan 2011; Subramanian et al. 2013).

\subsection{Linear growth/decay rates and frequencies}

We measured the linear growth rates, decay rates, and frequencies, in the small amplitude regime, for a range of heater powers. In the same framework, Provansal et al. (1987) described the transient behaviour of the wake of a circular cylinder above and below the critical Reynolds number for vortex shedding, and experimentally measured linear growth rates and frequencies.

To obtain the growth rates and frequencies at heater power $P$, the heater power was initially set just below that of the Hopf point, $P_{H}-\epsilon$, where $\epsilon=15$ Watts. This value of $\epsilon$ was the minimum such that triggering due to environmental disturbances was avoided (Juniper 2011). The heater power was then increased abruptly to $P$. Oscillations grew to a limit-cycle (figure 3a) and the linear regime was identified as that in which the oscillations grew exponentially (figure 3c). 

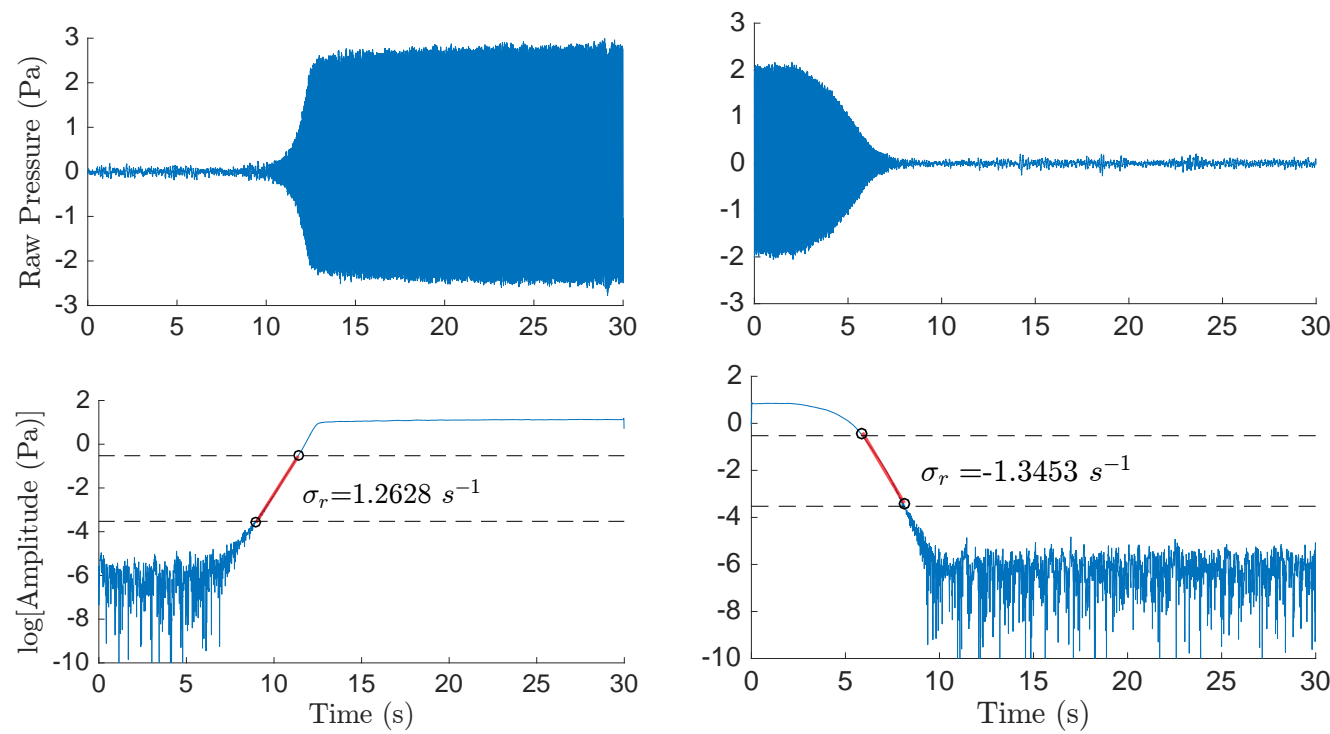

Figure 3. Top: Transient pressure signals obtained by applying step changes to the heater power input. (a) Growth, from $P_{H}-\epsilon$ to $P=223 \mathrm{~W}$. (b) Decay, from $P_{f}+\epsilon$ to $P=140 \mathrm{~W}$. Bottom: Amplitude of the filtered pressure signals obtained from the Hilbert transform. The linear growth (c) and linear decay (d) regions are represented by the red lines fitted between the noise floor and the point where nonlinear effects become important.

To obtain the decay rates and frequencies at power $P$, the heater power was initially set just above that of the fold point, $P_{F}+\epsilon$. It was then decreased abruptly to $P$. Oscillations decayed to a stable fixed point (figure $3 \mathrm{~b}$ ) and the linear regime was identified as that in which the oscillations decayed exponentially (figure $3 \mathrm{~d}$ ).

The procedure described above to obtain growth and decay rates was repeated for a range of heater powers $P$ above the Hopf point and below the fold point, respectively. The instantaneous amplitude, $A(t)$, and phase, $\phi(t)$, of the pressure signal was extracted with the Hilbert transform (Schumm et al. 1994). A bandpass Butterworth filter was applied to the raw pressure signal to reduce the noise, enabling clean regions of linear growth and decay to be identified. The linear growth and decay regions were defined as the regions between the noise floor, $A>\exp (-3.38) \approx 0.03 \mathrm{~Pa}$, and the amplitude threshold where nonlinear effects become important, $A>\exp (-0.52) \approx 0.59 \mathrm{~Pa}$. In the linear regime, the growth/decay rate is $\sigma_{r}=d(\log A) / d t$ and the frequency is $\sigma_{i}=d \phi / d t$. Within the linear growth and decay regions defined above, constant growth/decay rates and frequencies were fitted to the data using linear regression, and the results are shown in figure 4 . Close to the critical power, $P_{c r i t}=P_{H}$, the growth rate and frequency can be approximated by

$$
\sigma \simeq \sigma\left(P_{\text {crit }}\right)+\left.\left[P-P_{\text {crit }}\right] \frac{d \sigma}{d P}\right|_{P_{c r i t}},
$$

where $\sigma=\sigma_{r}+i \sigma_{i}$, as shown in figure 4 by the fitted lines. Notice that $\sigma_{i}$ is expressed in $\mathrm{Hz}$. For the baseline case, $P_{c r}=181.49 \mathrm{~W}$, and $\sigma_{r}\left(P_{\text {crit }}\right)=0$. It was found that $\sigma_{i}\left(P_{\text {crit }}\right)$ $=183.81 \mathrm{~Hz}$, and the gradients are $d \sigma_{r} / d P=0.0236$ and $d \sigma_{i} / d P=0.0211(\mathrm{Ws})^{-1}$. 

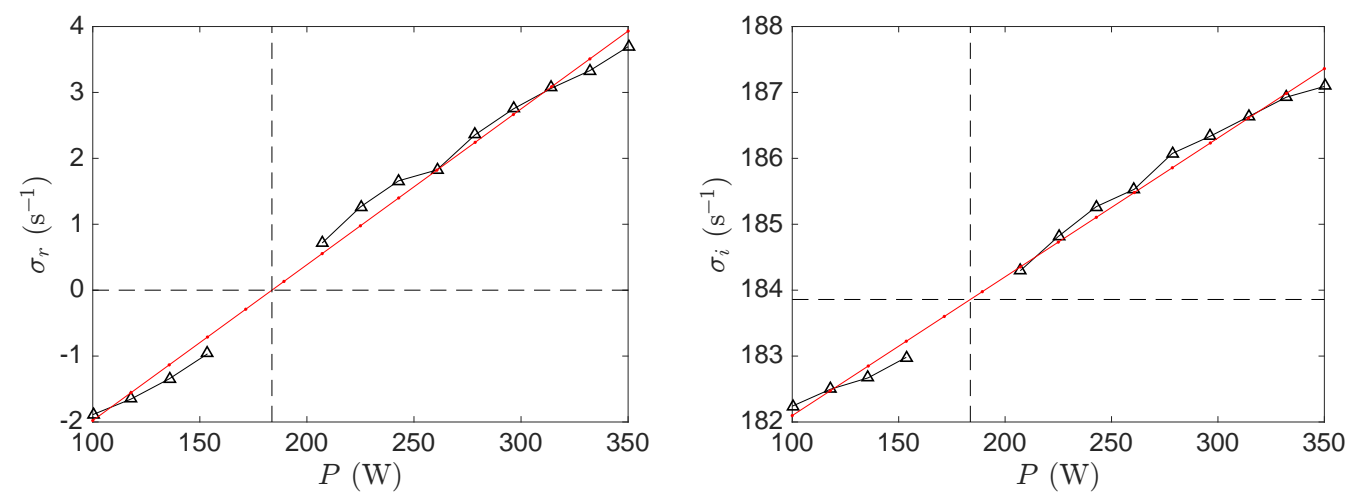

FiguRE 4. Linear stability characteristics for baseline case as a function of the primary heater power: (a) growth/decay rate, $\sigma_{r}$; (b) frequency, $\sigma_{i}$. Experimental data (symbols) and linear fit (red line).

\section{Passive control via a drag device}

In this section, we examine the change of the linear stability characteristics of the system, quantified as shifts in the growth rate and oscillation frequency, that was caused by the introduction of a passive drag device. The eigenvalue shift defined in the theoretical analysis of Magri \& Juniper (2013) was obtained here experimentally as

$$
\delta \sigma\left(x_{c}, P\right)=\sigma_{c}\left(x_{c}, P-P_{c r i t, c}\right)-\sigma_{0}\left(P-P_{c r i t, 0}\right) .
$$

The subscript $c$ corresponds to the case with the passive drag device installed and the subscript 0 to the baseline case. A procedure similar to the one followed in $\S 3$ was followed to obtain $\sigma_{c}$, when the drag device was placed at $x_{c} / L$ from 0.05 to 0.95 .

\subsection{Critical power}

For each position $x_{c}$ of the drag device, a bifurcation diagram similar to figure 2 was obtained enabling the Hopf and fold points to be located. These are shown in figure 5 . The minimum forward and backward path critical powers correspond to the drag device being positioned at $x_{c} / L=0.55$, and the maxima towards the two ends of the tube. To a first order, the sensitivity of the growth rate around the critical baseline power is proportional to the change of the critical power in the presence of a control device. An estimate of the sensitivity of the growth rate can be obtained from the critical power shift. However, in this study we measured it directly, as described next.

\subsection{Growth rate shift}

The growth rates and frequencies were measured experimentally for various heater powers, $P$, and axial positions, $x_{c}$, of the drag device. A comparison with the baseline at the same heater power $\mathrm{P}$ gives the growth rate and frequency shift, $\delta \sigma$, caused by the passive drag device. Time series were measured to obtain the growth/decay rates and are plotted for $x_{c} / L=0.4$ in figure 6 . Data were acquired for various axial locations, $x_{c} / L$; for brevity, only one case is shown. A map of the growth and decay rates as a function of heater power and $x_{c} / L$ is shown in figure $7 \mathrm{a}$, and a map of the shift in growth rate relative to the baseline is shown in figure 7b. In figure 8 we qualitatively compare the experimentally measured growth rate shift with the theoretical predictions from Magri \& Juniper (2013). Each experimental curve corresponds to a different heater power, $P$. The mean of these is shown as a solid black line. In agreement with the predictions, the 


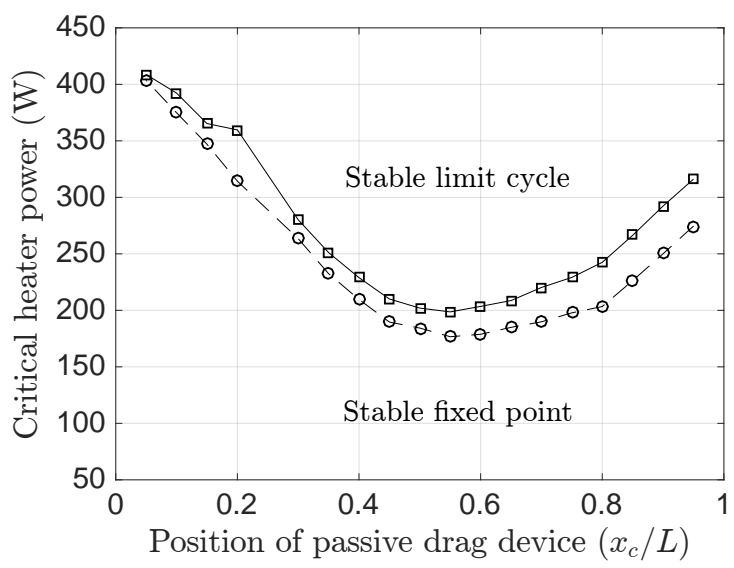

Figure 5. Stability regions as a function of the drag device position. Forward path critical powers: Hopf points (squares); backward path critical powers: fold points (circles). A bistable region exists between the two lines.
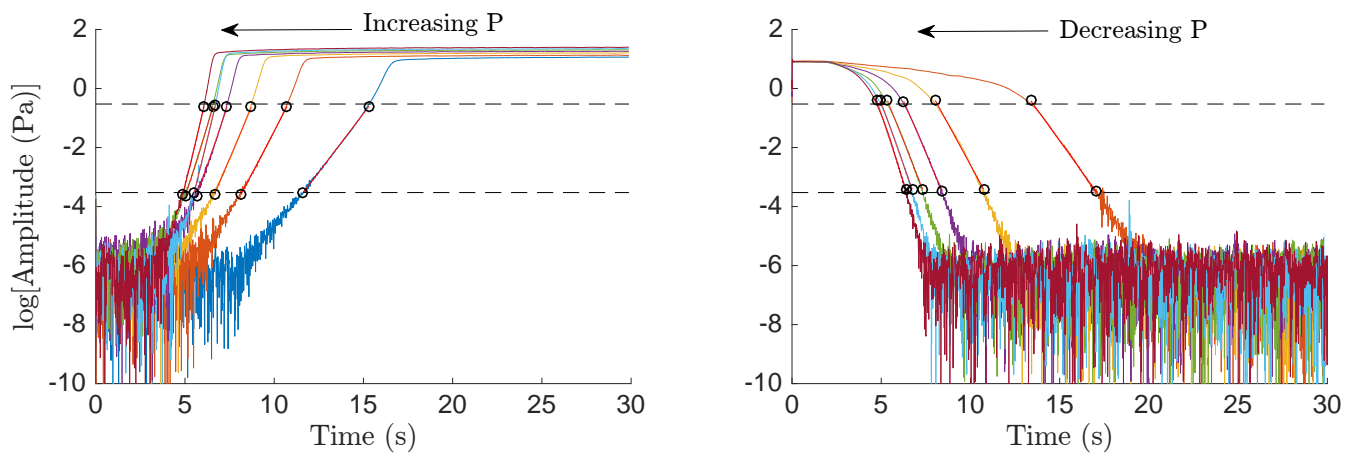

Figure 6. (a) Growth rates and (b) decay rates, $\sigma_{r, c}$, obtained with the control drag device positioned at $x_{c} / L=0.4$. Each curve represents the amplitude response for a different departure size above or below the Hopf or fold point.

largest changes in growth and decay rates occur when the drag device is positioned at the ends of the Rijke tube: $x_{c} / L=0.05$ and 0.95. Magri \& Juniper (2013) described this type of passive control as the feedback mechanism which is proportional to velocity, and which forces the momentum equation, in the same direction as the velocity. The effect of this feedback mechanism on the growth rate was theoretically predicted by the first diagonal component of the structural sensitivity tensor, $S_{11}$. Because drag is in the opposite direction to the velocity, we plot $-\delta \sigma_{r}$ on the vertical axis.

\subsection{Frequency shift}

The frequency shift in the presence of the drag device was also measured and is shown in figure 9b. Magri \& Juniper (2013) predict that the frequency shift should be two orders of magnitude smaller than the growth rate shift and here we measure it to be of the same order. As expected, in the experiment we noticed a non-negligible increase of the mean air temperature downstream of the heater. The increased temperature is mainly due to the increased critical power required for the transition of the system, $P_{c r i t, c}$, when the control device is introduced, see figure 5. Magri \& Juniper (2013) examined the sensitivity of the system to changes in the heat release parameter, which in the experimental setup 

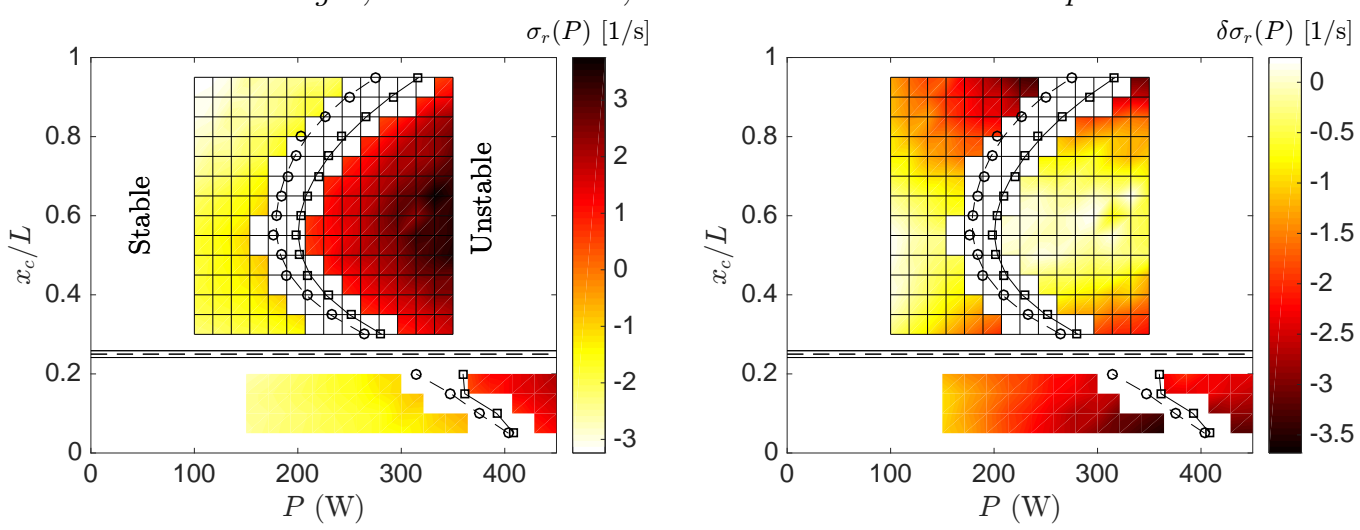

FIGURE 7. (a) Growth rate and (b) growth rate shift experimentally obtained as a function of power supplied to the heater and position of the drag device. Forward path critical powers (squares) and backward path critical powers (circles). The primary heater was located at $x_{c} / L=0.25$.
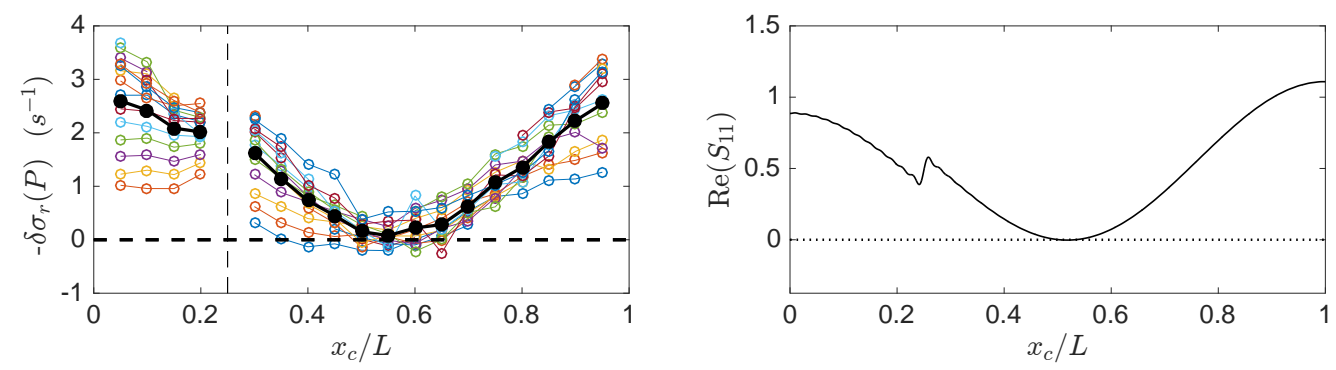

Figure 8. Sensitivity of the growth rate to a drag device. (a) Experimental results and (b) theoretical predictions by Magri \& Juniper (2013). Each experimental curve corresponds to different primary heater powers; the average sensitivity is also shown (solid circles).

can be linked to the power supplied to the heater. They found that a variation in the heat release parameter, here $P_{\text {crit }}$, has a much greater effect on the frequency than on the growth rate. For a theoretical justification, see Magri \& Juniper (2013), pg. 197.

The mean temperature deviation due to the increase of $P_{\text {crit }}$, which is not captured in the Magri \& Juniper (2013) model, changes the speed of sound and the frequency of the instability. If the mean air temperature is taken to be the outlet air temperature, which was measured during the experiment, then an estimate of the expected frequency of the fundamental ( $1 / 2$ wavelength) mode is given by:

$$
f=\left[\frac{2\left(L_{u}+0.61 D\right)}{c_{u}}+\frac{2\left(L_{d}+0.61 D\right)}{c_{d}}\right]^{-1},
$$

where $L_{u}$ and $c_{u}$ are the length of tube and speed of sound upstream of the heater, $L_{d}$ and $c_{d}$ are downstream quantities, and $0.61 D$ is the end correction, where $D$ is the tube diameter (Rienstra \& Hirschberg 2006). Figure 10 compares the frequency shift calculated from (4.2) with that measured in the experiments. The two are close and show the same features. This indicates that the frequency shift due to changes in the mean conditions greatly exceeds the expected frequency shift due to feedback from the drag device. Therefore, it can be inferred that the latter is minimal within the experimental error of the measurements. 

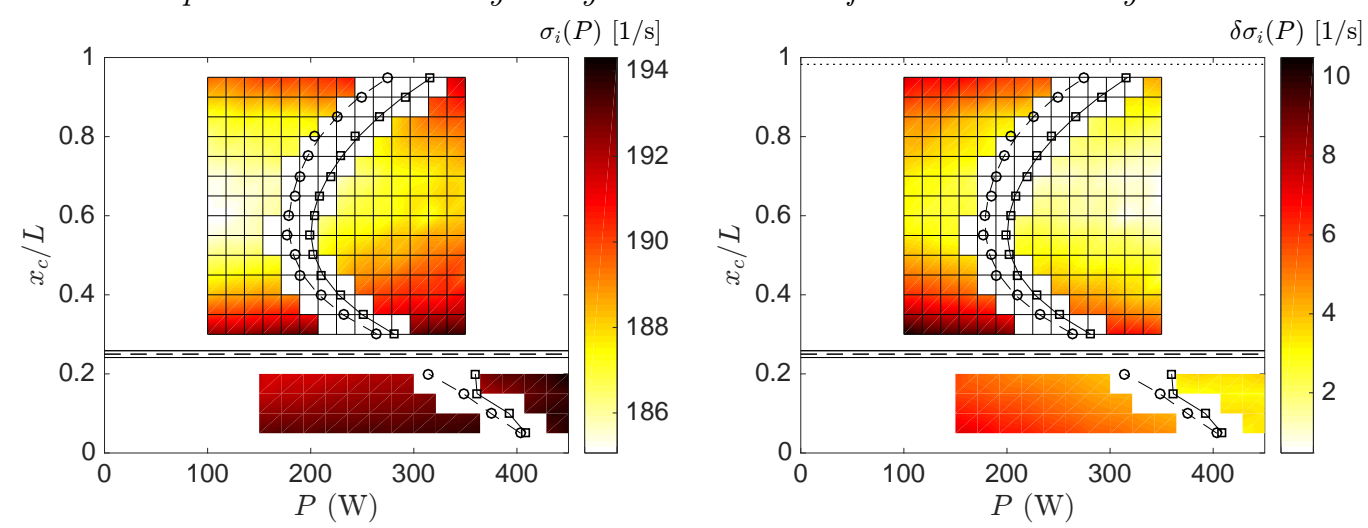

FiguRE 9. (a) Frequency and (b) frequency shift experimentally obtained as a function of power supplied to the heater and position of the drag device. Forward path critical powers (squares) and backward path critical powers (circles).
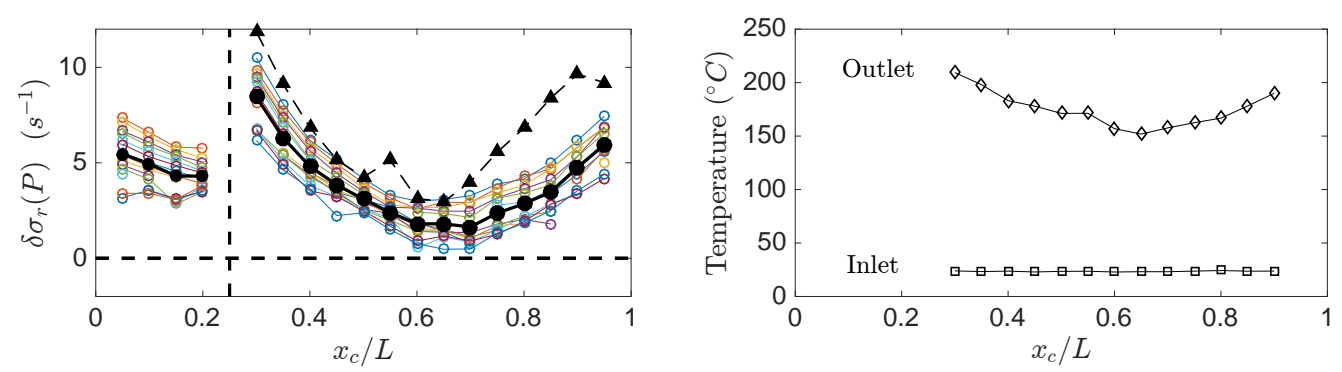

FiguRE 10. (a) Sensitivity of the frequency to a drag device. Experimental results for various primary heater powers (open circles) and average (solid circles). An estimate of the frequency change (solid triangles) due to variations in the outlet temperature shown in (b).

\section{Conclusions}

The control of thermoacoustic oscillations is a significant problem in the development of clean and efficient combustion systems. Previous work by Magri \& Juniper (2013) has shown that adjoint-based methods are a computationally efficient tool by which optimal passive techniques can be developed and implemented to control thermoacoustic oscillations. We show that sensitivity analysis can be performed experimentally on a vertical Rijke tube to determine how sensitive the growth rates and frequencies are to passive feedback control in the form of a drag device. We measure the shift in growth and decay rates and frequency and compare our results with those obtained by Magri \& Juniper (2013). We provide experimental evidence that supports adjoint-based methods being applied to determine the most efficient methods of passive control in thermoacoustic systems.

The authors would like to thank Luca Magri (Department of Engineering, University of Cambridge, UK) for invaluable discussions and comments on this paper. This work was supported by the European Research Council through Project ALORS 2590620.

\section{REFERENCES}

Candel, S. M. 2002 Combustion dynamics and control: Progress and challenges. Proc. Combust. Inst. 29, 1-28. 
Crocco, L., Mitchell, C. E. \& Sirignano, W. A. 1969 Nonlinear longitudinal instability in rocket motors with concentrated combustion. Comb. Sci. Tech. 1, 33-64.

Culick, F. E. C. 1988 Combustion Instabilities in Liquid-Fuelled Propulsion Systems. Tech. Rep. 430.

Dowling, A. P. \& Morgans, A. S. 2005 Feedback Control of Combustion Oscillations. Annu. Rev. Fluid Mech. 37 (1), 151-182.

JuniPER, M. P. 2011 Triggering in the horizontal Rijke tube: non-normality, transient growth and bypass transition. J. Fluid Mech. 667, 272-308.

Katto, Y. \& SAJiki, A. 1977 Onset of oscillation of a gas-column in a tube due to the existence of heat-conduction field: a problem of generating mechanical energy from heat. Bulletin of JSME 20 (147), 1161-1168.

Lieunen, T. C. \& YANG, V. 2005 Combustion instabilities in gas turbine engines: operational experience, fundamental mechanisms and modeling. Progress in Astronautics and Aeronautics .

Luchini, P. \& Bottaro, A. 2014 Adjoint Equations in Stability Analysis. Annu. Rev. Fluid Mech. 46 (1), 493-517.

MAGRI, L. \& Juniper, M. P. 2013 Sensitivity analysis of a time-delayed thermo-acoustic system via an adjoint-based approach. J. Fluid Mech. 719, 183-202.

Magri, L. \& Juniper, M. P. 2014 a Adjoint-based linear analysis in reduced-order thermoacoustic models. Int. J. Spray. Combust. 6 (3), 225-246.

Magri, L. \& Juniper, M. P. $2014 b$ Global modes, receptivity, and sensitivity analysis of diffusion flames coupled with duct acoustics. J. Fluid Mech. 752, 237-265.

MARIAPPAN, S. 2011 Theoretical and Experimental Investigation of the Non-Normal Nature of Thermoacoustic Interactions. PhD Thesis, Indian Institute of Technology, Madras.

Matveev, K. 2003 Thermoacoustic instabilities in the Rijke tube: experiments and modeling. $\mathrm{PhD}$ Thesis, California Institute of Technology.

McManus, K. R., Poinsot, T. \& Candel, S. M. 1993 A review of active control of combustion instabilities. Prog. Energy Combust. Sci. 19, 1-29.

Provansal, M., Mathis, C. \& Boyer, L. 1987 Bénard-von Kármán instability: transient and forced regimes. J. Fluid Mech. 182, 1-22.

Raun, R. L., Beckstead, M. W., Finlinson, J. C. \& Brooks, K. P. 1993 A Review of Rijke Tubes, Rijke Burners and Related Devices. Prog. Energ. Combust. 19, 313-364.

Rayleigh, J. W. S. 1878 The Explanation of Certain Acoustical Phenomena. Nature (London) 18, 319-321.

Rienstra, S. W. \& Hirschberg, A. 2006 An Introduction to Acoustics.

RiJke, P. L. 1859 On the vibration of the air in a tube open at both ends. Phil. Mag. 17, $419-422$.

SAito, T. 1965 Vibrations of Air-Columns Excited by Heat Supply. The Japan Society of Mechanical Engineers 8 (32).

Schmid, P. J. \& Brandt, L. 2014 Analysis of Fluid Systems: Stability, Receptivity, Sensitivity. App. Mech. Rev. 66 (2), 1-21.

Schumm, M., Berger, E. \& Monkewitz, P. A. 1994 Self-excited oscillations in the wake of two-dimensional bluff bodies and their control. J Fluid Mech. 271, 17-53.

Sipp, D., Marquet, O., Meliga, P. \& Barbagallo, A. 2010 Dynamics and control of global instabilities in open-flows: a linearized approach. Appl. Mech. Rev. 63 (3), 030801.

Sreenivasan, K. R., Raghu, S \& Chu, Boa-Teh 1985 The Control of Pressure Oscillations in Combustion and Fluid Dynamical Systems. AIAA Shear Flow Cont. Conf. pp. 1-10.

Subramanian, P., Sujith, R. I. \& Wahi, P. 2013 Subcritical bifurcation and bistability in thermoacoustic systems. J Fluid Mech. 715, 210-238. 